\title{
Distinguish Coexistence of Nanoemulsion and Liposome in Propofol by Cryogenic Transmission Electron Microscopy (cryo-TEM)
}

Yong $\mathrm{Wu}^{1}$, Peter Petrochenko ${ }^{1,2}$, Ja Hye Myung ${ }^{1,2}$, Soumyarwit Manna ${ }^{1,2}$, Bonhye Koo ${ }^{1,2}$, Stephanie Choi $^{2}$, Darby Kozak ${ }^{2}$, Jiwen Zheng ${ }^{1}$

1. Division of Biology, Chemistry and Materials Science, Office of Science and Engineering Laboratories, Center for Devices and Radiological Health, Food and Drug Administration, Silver Spring, MD, United States

2. Division of Therapeutic Performance, Office of Research Standards, Office of Generic Drugs, Center for Drug Evaluation and Research, Food and Drug Administration, Silver Spring, MD, United States

Generic drug products must demonstrate pharmaceutical equivalence and bioequivalence to the reference listed drug (RLD) to gain FDA approval. Even with qualitative/quantitative (Q1/Q2) sameness to the RLD, bioequivalence for complex products may not be ensured. Slight variations in manufacturing processes and/or formulation steps may give rise to differences in size, shape, composition, agglomeration/aggregation of formulation components, which may affect drug release, clearance, bioavailability and ultimately product bioequivalence. Therefore, comparative physicochemical characterization studies (Q3), such as evaluation of morphological properties provide evidence demonstrating generic and RLD product sameness that supports bioequivalence determination.

The morphology of nanoscale drug products may significantly affect their formulation stability and dissolution rate, thus impacting the safety and efficacy of these products. Therefore, reliable and suitable characterization techniques are required to demonstrate comparable morphology. Electron microscopy provides direct high-resolution imaging of emulsion and liposome particles compared to traditional optical techniques. Cryogenic transmission electron microscopy (cryo-TEM) can effectively preserve the native structure of samples in a frozen hydrated state and provide an accurate representation of complex RLDs and generic drug products in nanometer scale.

Propofol, an intravenous anesthesia drug substance, is barely soluble in water and is therefore formulated as an oil-in-water emulsion. Although the RLD product was approved in 1989 and 1996, followed by approval of several generic products, there is still limited information on the morphological properties of this product.

In our results, cryogenic transmission electron microscopy (cryo-TEM) micrographs revealed the RLD and 3 generic propofol products are mixtures comprising emulsions, liposomes and emulsion-liposome combined structures (Figure 1). All evaluated propofol products possess a heterogeneous size distribution of oil globules and liposomes, with the majority of oil globules in size range of 100-200 nm. Liposomes in RLD and generic propofol formulations include small unilamellar vesicles $(<100 \mathrm{~nm})$ and large unilamellar vesicles $(100-1,000 \mathrm{~nm})$. Most of the evaluated products comprise $70-80 \%$ of emulsions and 20-30\% liposomes, while some formulation shows a higher fraction of liposomes. Overall, our findings outline a potential method for identifying individual components (e.g. nanoemulsion and liposomes) with complex formulations, in their native states. Such accurate and reliable physicochemical characterization techniques are expected to offer prospective guideline for manufacturing and design control of complex drug formulations. 


\section{References:}

[1] Y Wu, et al, International journal of pharmaceutics 505 (2016), p. 167.

[2] Y Wu, et al, Microscopy and Microanalysis 20 (2014), p. 1348.

[3] Y Wu, et al, Microscopy and Microanalysis 20 (2014), p. 1228.

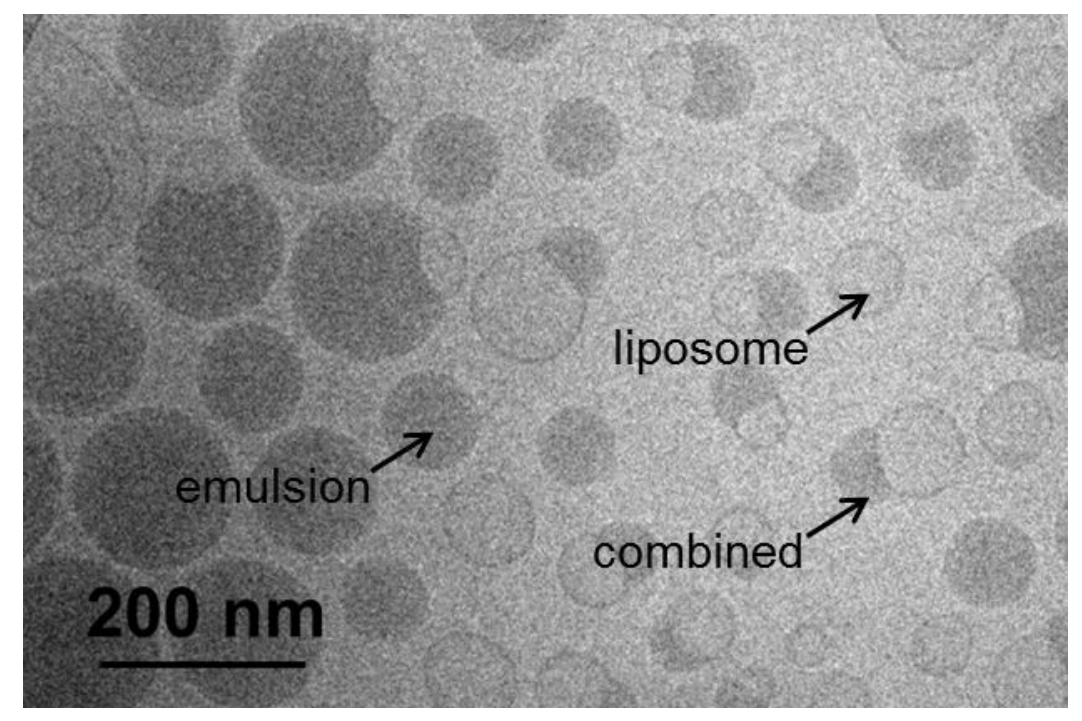

Figure 1. Representative cryo-TEM micrograph of propofol showing it contains emulsion, liposome and combined emulsion-liposome structure. 